\title{
La aplicación de la extensión territorial del Derecho de la Unión Europea
}

\section{The Implementation of Territorial Extension in European Union Law}

\author{
Ana Cristina Gallego Hernández \\ Profesora de Derecho Internacional Público y Relaciones Internacionales \\ Centro Universitario San Isidoro (UPO) \\ aagallegoo@gmail.com
}

Sumario: I. Introducción.-II. Extraterritorialidad del Derecho tanto dentro, como fuera de las fronteras de la UE.-III. La Extensión Territorial de las normas en la UE.-IV. Confirmación de la teoría de la Extensión Territorial por el TJUE.-V. Conclusiones.

Resumen: La aplicación extraterritorial del derecho es congruente con el principio de respeto a la soberanía y, correlativamente con el principio de no intervención, cuando su ejercicio se fundamente en los intereses generales y siempre que prime la proporcionalidad. A pesar de que la UE nunca ha sido una completa defensora de la extraterritorialidad, especialmente tras la última gran crisis económica europea y mundial, comienza a redoblarse el ejercicio por parte de la referida organización, a través de la extensión territorial, la cual permite controlar aquellas conductas que, aunque se lleven a cabo en el extranjero, repercutan en los intereses generales de la UE. La doctrina se ha encargado de conceptualizar la modalidad de la extensión territorial, no obstante, la confirmación de la teoría no llega hasta que el TJUE se pronuncia sobre la Directiva 2008/101/CE del Parlamento Europeo y del Consejo, de 19 de noviembre de 2008, llamada Directiva de Aviación, en 2011. TJUE.

Palabras clave: Extraterritorialidad, extensión territorial, principio territorial,

Abstract: The extraterritorial application of law is consistent with the principle of respect for sovereignty, and correlatively with the principle of nonintervention, when its exercise is based on general interests and when there is proportionality. Despite the fact that the EU has never been a complete defender of extraterritoriality, especially after the last great world economic crisis, the exercise by the aforementioned organization begins to double. Territorial extension is used to control those behaviors that, although carried out abroad, have an impact on the overall EU interests. The doctrine has been in charge of conceptualizing the 
modality of the territorial extension. However, it was the CJEU that confirmed this when it pronounced itself about Directive 2008/101/EC of the European Parliament and of the Council of 19 November 2008, called the EU Aviation Directive, in 2011.

Keywords: Extraterritoriality, territorial extension, territorial principle, CJEU.

\section{Introducción}

Tradicionalmente, la autoridad de un Estado ha regulado exclusivamente aquellos eventos acaecidos en su propio territorio, es decir, en el interior de sus fronteras. No obstante, a lo largo del siglo Xx, el concepto de territorialidad se ha ido expandiendo, y ha ido incluyendo también a aquellas conductas desarrolladas fuera de las fronteras del Estado en cuestión, pero con efectos en él ${ }^{1}$. De esta forma, surge una corriente en coherencia con el Derecho Internacional ${ }^{2}$, sobre la aplicación extraterritorial del Derecho, tanto a todas las conductas ocurridas en el propio territorio, como a aquellas conductas extranjeras con efectos sobre el Estado soberano.

En lo que respecta a las organizaciones internacionales, igualmente, se ha ido desarrollando una aplicación extraterritorial ${ }^{3}$, relativamente similar al de los sujetos principales de Derecho Internacional. En concreto, la Unión Europea - en adelante UE-, como organización internacional de integración, regional y de fines generales, ostenta un complejo y extenso derecho originario y derivado cuya aplicación se está viendo amplificada, en ocasiones hasta de manera global.

1 «"Territoriality" and "extraterritoriality" (...) are legal constructs», Hannah Buxbaum, «Territory, Territoriality, and the Resolution of Jurisdictional Conflict», American Journal of Comparative Law, 57, 3 (2009): 636.

2 Ello se fundamenta de manera general sobre el principio de igualdad soberana y, en concreto, en la regla de que cada Estado goza de los derechos inherentes a la plena soberanía, Resolución 2625 (XXV) de la Asamblea General de Naciones Unidas.

3 «Una estricta aplicación de ese territorialismo es incompatible con numerosas situaciones en las que están presentes elementos de extraterritorialidad y en las que una misma conducta es susceptible de producir efectos jurídicos en distintas partes del territorio de la Unión. La construcción de una Europa sin fronteras, con su corolario de aproximación de los diferentes ordenamientos jurídicos nacionales, comprendidos los penales, presupone que los Estados implicados se inspiran en los mismos valores. Y es aquí, en el ámbito de los valores, donde el principio que se examina alcanza todo su sentido». Conclusiones del Abogado General Ruiz-Jarabo Colomer, presentadas el 19 de septiembre de 2002, Asuntos C-187/01 y C-385/01. 
Autores como la Profesora Scott ${ }^{4}$ consideran que la aparición de estos nuevos desencadenantes en la legislación europea está vinculada a la crisis financiera ${ }^{5}$ de los últimos años. No obstante, en mi opinión, esto no puede ser mal interpretado, en el sentido de que en situación de inestabilidad económica, la UE haya preferido anteponer el interés puramente económico, sino que las medidas adoptadas buscan proteger los intereses europeos de los riesgos financieros, proteger la estabilidad e integridad de los mercados financieros de la UE y evitar que la autoridad de la UE se vea perjudicada tras la evasión normativa ${ }^{6}$. Por ello, la UE, cada vez más, utiliza la aplicación extraterritorial de su derecho y, en concreto la extensión territorial, para controlar las conductas que, aunque se lleven a cabo en el extranjero, repercutan en sus propios intereses.

En este sentido, los objetivos específicos que este trabajo persigue son esencialmente tres: poder justificar la garantía del principio territorial, conocer cómo en las distintas esferas se aplica la extraterritorialidad del derecho por parte de los Estados miembros y de la propia UE y, analizar la aplicación de la extensión territorial por parte de la UE. Para ello, dada la complejidad del análisis que propongo, el cual requiere tener en cuenta aspectos confusos, me voy a permitir utilizar una metodología científica sistémica que me dé la oportunidad de abordar esa ardua tarea teniendo en cuanta todos los sectores del Derecho implicados, bien como ordenamientos jurídicos completos, Derecho Internacional Público, Derecho de la UE, sea primario o derivado, ordenamientos internos estatales, bien de Estados Miembros de la UE o No Miembros que estén implicados, Derecho Internacional Privado, sectores jurídicos complementarios como Derecho del Mar, Derechos Humanos, etc.

\section{Extraterritorialidad del derecho tanto dentro, como fuera de las fronteras de la UE}

Partiendo de la tesis de que la legítima aplicación extraterritorial del derecho es congruente con el principio de respeto a la soberanía y, correlati-

${ }^{4}$ «Consequently, recourse to these novel triggers serves to expand the global reach of EU law, bringing within its scope behaviour that would previously have been regulated exclusively by other States». Joanne Scott, «New EU Extraterritoriality», The Common Market Law Review, 51, 5 (2014): 1363.

5 «It is noteworthy that the emergence of these novel «extraterritorial» triggers in EU law is linked to the global financial crisis. They form part of an understandable legislative backlash against domestic, EU and global financial markets characterized by «light touch» regulation and the socialization of huge private losses through bail-out mechanisms, the costs of which are borne by the taxpayer. One impact of the global financial crisis and of the associated». Ibid., p. 1345.

${ }^{6}$ Ibid., p. 1364. 
vamente con el principio de no intervención ${ }^{7}$, su ejercicio se fundamenta, adicionalmente, en la proporcionalidad y en la consecución de intereses generales ${ }^{8}$. La postura que adopta la UE, para que así se garantice el principio territorial ${ }^{9}$ y se justifique la extraterritorialidad, es que es suficiente que los efectos territoriales de una determinada acción - tanto fuera como dentro de las fronteras -, puedan identificarse con la propia $\mathrm{UE}^{10}$. En este sentido, la aplicación extraterritorial de una norma, requerirá un vínculo jurisdiccional suficiente a través de competencias ratione materiae, loci, personae o temporis ${ }^{11}$, lo cual - en el escenario planteado- será controlado por los órganos nacionales correspondientes y por la Comisión, el Parlamento Europeo y, especialmente, el Tribunal de Justicia de la Unión Europea - en adelante TJUE- .

7 A pesar de que en ocasiones se pueden generar situaciones tensas o, como lo califica inéditamente el Profesor Fernández Sánchez, de «soberanía desafiante». Pablo Antonio Fernández Sánchez, «La soberanía poliédrica», Soberanía del Estado y derecho internacional: homenaje al profesor Juan Antonio Carrillo Salcedo, (Pérez Vera, E., Rodríguez Carrión, A.) (2005) Universidad de Málaga: 609-611.

8 Justificado por el aumento de la protección y el compromiso en la comunidad internacional institucionalizada. Jan Wouters, Cedric Ryngaert, «Litigation for Overseas Corporate Human Rights Abuse in the European Union: The Challenge of Jurisdiction», George Washington International Law Review 40, 4, (2009): 956.

En el mismo sentido, cita la profesora Buxbauma a «Karl Meessen asserted on the basis of the nonintervention principle that international law affirmatively required some form of interest balancing: that "the governmental interests of the regulating state will suffice to justify the exercise of jurisdiction, unless the governmental interests of the foreign state significantly outweigh them."» Hannah Buxbaum, «Territory, Territoriality, and the Resolution of Jurisdictional Conflict», American Journal of Comparative Law, 57, 3 (2009): 657.

${ }_{9} \mathrm{El}$ principio de territorio no es absoluto y caben múltiples excepciones de conformidad al Derecho Internacional que permite no tener que dividir el mundo en compartimientos estancos. No obstante, esto implica flexibilizar la vinculación entre soberanía, territorio y jurisdicción para que cada Estado (u organización internacional, en su caso) pueda preservar sus intereses legítimos fruto de las interacciones sociales, sin que se delimite de manera estricta y geográficamente el territorio en cuestión.

10 «What is relevant is whether (substantial) conduct or effects could be located on the territory»-objective territoriality - , or «territorial effects could be identified, even absent any territorial conduct, or conversely that territorial conduct could be identified, even absent any territorial effects» - subjective territoriality - . Cedric Ryngaert, «EU Trade Agreements and Human Rights: From Extraterritorial to Territorial Obligations», International Community Law Review, 20 (2018): 384.

11 «Para que el tribunal pueda conocer de un caso que se somete ante él, debe verificar que posee competencia en razón de las partes que intervienen en el procedimiento (ratione personae); en razón de la materia objeto de la controversia (ratione materiae); en razón del momento en que el Estado demandando ha ratificado el tratado (ratione temporis); y, finalmente, en razón del lugar donde ocurrieron los hechos (ratione loci)». Juan Pablo Pérez-León, Patrick Wieland, «La actuación extraterritorial del Estado: re-examinando el ámbito ratione loci desde el Derecho Internacional contemporáneo», Ius et Veritas, 34 (2007): 278. 
Así las cosas, el fenómeno, un tanto peculiar, que se desarrolla en el seno de la UE viene fundamentado por la cesión ${ }^{12}$ - relativa - de competencias soberanas, lo cual justifica que la propia organización internacional pueda ejercitar la aplicación extraterritorial dentro y fuera de sus fronteras. A pesar de que, la verdadera soberanía, no podemos olvidarlo, siempre será ejercida, en última instancia, por los propios Estados Miembros.

En este sentido, es una realidad que la UE en los últimos años es cada vez más partidaria de la extraterritorialidad de la norma, especialmente a través de la figura de la extensión territorial. No obstante, al existir una interrelación particular entre distintos sujetos internacionales en el escenario objeto de estudio y al desarrollarse distintas vías para exigir los referidos efectos extraterritoriales, a continuación, se bosquejan diversas modalidades, las cuales van definiendo la postura europea.

La extraterritorialidad, desde la perspectiva más básica, consiste en la aplicación de normas y en el impulso de políticas propias más allá del propio territorio al que concierne. No obstante, debido a que la extraterritorialidad (justificada y aceptada) es una excepción que confirma la prohibición de injerencias en los asuntos internos, la proyección práctica no solo es ambigua, sino que presenta límites y condicionantes.

Generalmente cuando se practica la aplicación extraterritorial por un tercer Estado, lo que este lleva a cabo es la aplicación directa de una norma fuera de su territorio, sin que la posibilidad se recoja en la misma. Dicho ejercicio se realiza en congruencia con el Derecho Internacional, por tanto, fundamentado en el principio de diligencia debida y sin que una conexión territorial justifique la acción referida ${ }^{13}$. Sin embargo, en la esfera de la UE, esta práctica no resulta común ${ }^{14}$, por eso para poder exigir extraterritorialmente ciertas actuaciones, la UE ha ido desarrollando y consolidando otra

12 «As is common knowledge, the Member States have constituted the EU mainly through the transfer of sovereign powers, on which the foundation of both original and derivative law is based. Therefore, with regard to Member States, the European legal system erects the cover under which all its domestic law is structured. Thus, the extraterritorial application of the rules of Internal Law of the European States must be implemented in accordance with the aforementioned Union Law». Ana Cristina Gallego Hernández, «Extraterritoriality within the framework of regional organizations: the case of the European Union», Hague Academy of International Law, (2019) pendiente de publicación.

${ }^{13}$ Evidentemente, sí que puede justificarse la aplicación fuera del territorio por otros tipos de nexos, como por ejemplo vinculación temporal o nacionalidad. Todo ello, teniéndose presente que la obligación asumida por el tercer Estado debe ser coherente con el Derecho Internacional y basada en la cooperación.

14 «The EU's aversion to extraterritoriality is not countered by a strong reliance on effects-based jurisdiction. Effects has reared its head quite recently, though as yet tentatively, in EU financial services regulation». Joanne Scott, «Extraterritoriality and Territorial Extension of EU Law», American Journal of Comparative Law, 62, 1 (2014): 95. 
modalidad de aplicación extraterritorial que es comúnmente conocida como extensión territorial, la cual será desarrollada en el apartado tercero.

Respecto a qué sujetos de derecho internacional pueden intervenir en la aplicación extraterritorial de derecho europeo, esquemática e inicialmente, se debe distinguir si la extraterritorialidad se desarrolla dentro del propio territorio de la UE, o bien, desde la propia organización hacia cualquier sujeto de la comunidad internacional ${ }^{15}$.

En lo que respecta al interior de la UE, como es de común conocimiento, cada Estado Miembro sin perjuicio de las competencias compartidas, sigue ejerciendo plenamente la soberanía sobre el resto de otras muchas esferas jurídicas y políticas, por lo que entre los propios Estados socios del mismo «club» pueden existir diferentes niveles de compromisos que acaben condicionando la aplicación extraterritorial de normas o disposiciones. O bien, en otras ocasiones, el posible ejercicio extraterritorial puede verse fundamentado en que, como Estados socios, se requiere la aplicación de una determinada disposición para salvaguardar el Derecho europeo que, de manera directa o indirecta, acaba en contradicción. En otras palabras y de manera práctica, cuando Rumanía impide la residencia del cónyuge de un nacional, porque el matrimonio entre dos personas del mismo sexo - celebrado en Bélgica - no tiene efectividad, por ser contrario al derecho interno del primero, se está actuando en contra del derecho europeo a circular y residir en el territorio de los Estados ${ }^{16}$. Para salvar dicha incongruencia, el TJUE ${ }^{17}$, a través de su interpretación, exige la aplica-

15 Esto es lo que llamo Extraterritorialidad «ad intra» y «ad extra» en mi trabajo: Ana Cristina Gallego Hernández, «Extraterritoriality within the framework of regional organizations: the case of the European Union», Hague Academy of International Law, (2019) pendiente de publicación.

${ }^{16}$ Directiva 2004/38/CE del Parlamento Europeo y del Consejo, de 29 de abril de 2004, relativa al derecho de los ciudadanos de la Unión y de los miembros de sus familias a circular y residir libremente en el territorio de los Estados miembros, DOUE 30.4.2004, L 158.

«Por lo que respecta a los requisitos de concesión de este derecho de residencia derivado, el Tribunal de Justicia ha destacado que no deben ser más estrictos que los establecidos por la Directiva 2004/38 para la concesión de tal derecho de residencia a un nacional de un tercer Estado, miembro de la familia de un ciudadano de la Unión que ha ejercido su derecho de libre circulación estableciéndose en un Estado miembro distinto de aquel del que es nacional, ya que esta Directiva debe aplicarse por analogía a la situación a la que se hace referencia en el apartado anterior de la presente sentencia (véanse, en este sentido, las sentencias de 12 de marzo de 2014, O. y B., C-456/12, EU:C:2014:135, apartados 50 y 61; de 10 de mayo de 2017, Chávez-Vílchez y otros, C-133/15, EU:C:2017:354, apartados 54 y 55, y de 14 de noviembre de 2017, Lounes, C-165/16, EU:C:2017:862, apartado 61)». Sentencia del Tribunal de Justicia (Gran Sala), de 5 de junio de 2018. Asunto C-673/16, párrafo 25.

17 «En cuanto a si este concepto incluye al nacional de un tercer Estado, del mismo sexo que el ciudadano de la Unión, con el cual ha contraído matrimonio en un Estado miembro de conformidad con el Derecho de ese Estado, debe recordarse ante todo que el concepto de «cón- 
ción, extraterritorialmente, de las consecuencias jurídicas del acto de unión matrimonial en Rumanía, a pesar de que el Estado ostenta la competencia para regular el estado civil de sus ciudadanos ${ }^{18}$.

Por otro lado, la extraterritorialidad ejercida por la UE más allá de sus fronteras es aquella en la que, como sujeto de Derecho Internacional, exige el cumplimiento de una conducta o de efectos extraterritoriales, de manera directa o bien, porque así ha sido estipulado previamente, a un tercer sujeto internacional. A este respecto, la UE como actor global, ejercita la extraterritorialidad ad extra de dos formas esencialmente distintas, aunque no es sencilla ni clara su distinción absoluta en la práctica. De manera tímida y porque resulta inevitable, a través de la aplicación directa del derecho de la UE fuera de su propio territorio, siempre en coherencia con los propios valores comunitarios ${ }^{19} \mathrm{y}$ sin que se justifique con un vínculo jurisdiccional ${ }^{20}$. Y, cada vez con más determinación, a través de la extensión territorial de una norma europea que desplegará efectos, también, fuera del territorio.

\section{La extensión territorial de las normas en la UE}

A pesar de que, como se viene comentando, la UE nunca ha sido una completa defensora de la extraterritorialidad, especialmente tras la última gran crisis económica europea y mundial, comienza a redoblarse el ejercicio por parte de la Organización que nos ocupa, a través de una modalidad prácticamente inédita, con el objetivo de afrontar problemas fronterizos y globales en aras del interés general.

yuge» en el sentido de la Directiva 2004/38 es neutro desde el punto de vista del género y puede, por tanto, incluir al cónyuge del mismo sexo del ciudadano de la Unión de que se trate. (...) «Es cierto que el estado civil de las personas, en el que se incluyen las normas relativas al matrimonio, es una materia comprendida dentro de la competencia de los Estados miembros, competencia que el Derecho de la Unión no restringe (véanse, en este sentido, las sentencias de 2 de octubre de 2003, García Avello, C-148/02, EU:C:2003:539, apartado 25; de 1 de abril de 2008, Maruko, C-267/06, EU:C:2008:179, apartado 59, y de 14 de octubre de 2008, Grunkin y Paul, C-353/06, EU:C:2008:559, apartado 16). Los Estados miembros disponen de ese modo de la libertad de contemplar o no el matrimonio entre personas del mismo sexo (sentencia de 24 de noviembre de 2016, Parris, C-443/15, EU:C:2016:897, apartado 59)». Sentencia del Tribunal de Justicia (Gran Sala), de 5 de junio de 2018. Asunto C-673/16, párrafos 35 y 37.

18 Artículo 277, apartados 1,2 y 4 del Código Civil Rumano.

19 Artículo 3.5 Tratado de la Unión Europea (TUE).

${ }^{20}$ La aplicación del derecho fuera del territorio sin que «depend on the existence of a territorial connection between a regulated activity and an EU Member State. [For example,] a measure that regulates the foreign conduct of EU citizens would be extraterritorial in this sense because its application posits nationality rather than territory as the relevant connection». Joanne Scott, « The global reach of EU law», EU law beyond EU borders: the extraterritorial reach of EU law (Marise Cremona, Joanne Scott), (Oxford, Oxford University Press, 2019): 22. 
La Extensión Territorial es una práctica que, principalmente, ha conceptualizado la Profesora Scott ${ }^{21}$, a través de la cual la UE, sin que se malinterprete como un atajo normativo, va encontrando el modo de poder ir reivindicando un alcance global de su legislación. Esto es, a diferencia de la extraterritorialidad «pura y dura», la extensión territorial no está ideada como un acceso directo para conseguir efectos jurídicos ${ }^{22}$ fuera del territorio, sino que, con su presencia cada vez más abundante, la Unión pretende defender los intereses generales de la organización y, correlativamente, de la comunidad internacional en general.

De manera puramente conceptual, la extensión territorial de una norma (por ejemplo, de una norma europea) consiste en la aplicación de una medida o disposición previamente regulada, que genera efectos jurídicos más allá de las fronteras (europeas) porque así está previsto en la norma en cuestión y, todo ello, porque se genera una conexión territorial entre ambos sujetos (en este caso, entre la UE y el otro sujeto en el que repercuta la obligación $)^{23}$.

A este respecto, para que la extensión territorial de una norma sea legítima, dicha obligación deberá perseguir objetivos globales, basados en el Derecho Internacional y, al mismo tiempo, vinculados necesariamente con los propósitos y el Derecho de la UE. Por tanto, el fin y objeto de la extensión territorial de una norma o medida europea será el de comprometer a los terceros Estados con obligaciones y estándares internacionales ${ }^{24}$ que se predigan en el seno, en este caso, de la UE. En definitiva, a través de la extensión territorial, la UE contribuye a la integración de los valores comunes y estimula a que exista mayor y mejor interrelación entre los sujetos que integran la comunidad internacional institucionalizada ${ }^{25}$.

${ }^{21}$ Ibid., p. 38.

22 Ibid., p. 41.

23 Así lo defino en el trabajo Ana Cristina Gallego Hernández, «Extraterritoriality within the framework of regional organizations: the case of the European Union», Hague Academy of International Law, (2019) pendiente de publicación.

24 «Frequently, the EU engages in the practice of territorial extension with a view to enforcing internationally agreed standards of conduct (international standards). Admittedly, it occasionally does so before the relevant international standards have entered into force, when the international standards are in a form that is not binding, and where they have been ratified by only a small number of states.99 There are nonetheless occasions when the EU self-consciously desists from extending the territorial reach of its measures because there are no corresponding international standards in place». Joanne Scott, «Extraterritoriality and Territorial Extension of EU Law», American Journal of Comparative Law, 62, 1 (2014): 112.

25 «While territorial extension (and to a lesser extent extraterritoriality) serves as an important tool to extend the global reach of EU law, it is not the only such tool. Indeed, assisted by the prospect of Brexit, the concept of the 'Brussels Effect' has garnered considerable attention. Building on David Vogel's earlier concept of the 'California Effect', Anu Bradford coined the 
Por estos motivos, como se ha adelantado, en los últimos años, la UE ha incrementado la aplicación de la extensión territorial a través de distintas normas que pretenden difundir ciertos aspectos de su Derecho y controlar las conductas en terceros Estados en cuestiones como medio ambiente ${ }^{26}$, transporte $^{27}$, salud $^{28}$, seguridad alimentaria ${ }^{29} \mathrm{o}$ servicios económicos y financieros ${ }^{30}$.

term the 'Brussels Effect' and drew a distinction between its de jure and de facto components». Joanne Scott, « The global reach of EU law», EU law beyond EU borders: the extraterritorial reach of EU law (Marise Cremona, Joanne Scott), (Oxford, Oxford University Press, 2019): 31.

26 A través de la Directiva 2009/30/CE del Parlamento Europeo y del Consejo de 23 de abril de 2009 por la que se modifica la Directiva 98/70/CE en relación con las especificaciones de la gasolina, el diésel y el gasóleo, se introduce un mecanismo para controlar y reducir las emisiones de gases de efecto invernadero, se modifica la Directiva 1999/32/CE del Consejo en relación con las especificaciones del combustible utilizado por los buques de navegación interior y se deroga la Directiva 93/12/CEE, la cual exige tener en cuenta las emisiones del combustible importado y su ciclo de vida, con el obejtivo de poder evaluar el cumplimiento del propósito de reducir la intensidad del combustible. Más reciente en el tiempo, la Directiva 2012/19/UE del Parlamento Europeo y del Consejo de 4 de julio de 2012 sobre residuos de aparatos eléctricos y electrónicos, a través de la cual se exige que las empresas vinculadas con la UE que recojan o traten aparatos electrónicos o eléctricos, cumplan con las condiciones mínimas de prevención, valorización y eliminación seguras de la UE.

27 Además de las normas citadas en este trabajo sobre la extensión territorial de condiciones en el transporte aéreo, existen otras vinculadas con el sector del transporte que presentan matices extraterritoriales. Por ejemplo: el Reglamento 391/2009 del Parlamento Europeo y del Consejo, de 23 de abril de 2009, sobre reglas y normas comunes para las organizaciones de inspección y reconocimiento de buques (ampliándose alguno de estos a los de terceros Estados); y el Reglamento 859/2011 de la Comisión, de 25 de agosto de 2011, que modifica en lo que atañe a la carga y al correo aéreos el Reglamento (UE) no 185/2010, por el que se establecen medidas detalladas para la aplicación de las normas básicas comunes de seguridad aérea y que, como su título indica, establece condiciones y pautas a empresas o terceros Estados cuando la carga o el correo entre o salga del territorio europeo.

${ }^{28}$ Reglamento 536/2014 del Parlamento Europeo y del Consejo, de 16 de abril de 2014, sobre los ensayos clínicos de medicamentos de uso humano, en el que se exige que se cumplan con los principios éticos en salud si se hacen ensayos clínicos fuera de la UE, con la finalidad de reintegrar los resultados.

29 Directamente, con el Reglamento 854/ del Parlamento Europeo y del Consejo, de 29 de abril de 2004, por el que se establecen normas específicas para la organización de controles oficiales de los productos de origen animal destinados al consumo humano. E indirectamente, el Reglamento 1005/2008, de 29 de septiembre de 2008 , por el que se establece un sistema comunitario para prevenir, desalentar y eliminar la pesca ilegal, no declarada y no reglamentada, de manera que se excluyen los productos de terceros Estados cuando no existe cooperación entre los sujetos internacionales.

${ }_{30}$ Como por ejemplo, el Reglamento 1060/2009 del Parlamento Europeo y del Consejo, de 16 de septiembre de 2009, sobre las agencias de calificación crediticia (a través del cual se exige dicha calificación crediticia cuando se opera con terceros Estados) o el Reglamento 648/2012 del Parlamento Europeo y del Consejo, de 4 de julio de 2012 , relativo a los derivados extrabursátiles, las entidades de contrapartida central y los registros de operaciones (que exige normas de efectos equivalentes para los terceros Estados que quieran operar en el mercado europeo). 
No obstante, como se indicaba en anteriores párrafos, la clave para distinguir si la aplicación extraterritorial de una norma se lleva a cabo de manera directa o bien a través de la extensión territorial, no siempre es clara y cristalina. En este sentido, en aras de poder identificar con mayor precisión cuando extiende más allá del propio territorio la obligatoriedad de una disposición o norma, a continuación, se enumeran las características principales, cuyo mérito identificativo principalmente debe atribuirse a la Prof. ${ }^{a} \operatorname{Scott}^{31}$.

El rasgo principal y más peculiar que, a mi juicio, nos permite reconocer que la extraterritorialidad se despliega mediante la extensión territorial, es que es requisito imprescindible que exista una conexión territorial. Esto quiere decir que debe ejercitarse cuando una conducta que tenga vinculación física o jurídica con el territorio del sujeto en cuestión. Evidentemente dicha conexión no se exigirá que sea continua, ni estática. Por lo que puede considerarse conexión territorial desde que un determinado buque ${ }^{32}$ o aeronave ${ }^{33}$ entre o salga del territorio de la UE, hasta que una empresa, Google Ireland Limited ${ }^{34}$, deba acatar la «Ley de Impuestos sobre la Publicidad» ${ }^{35}$

31 Joanne Scott, «Extraterritoriality and Territorial Extension of EU Law», American Journal of Comparative Law, 62, 1 (2014): 90. Joanne Scott, « The global reach of EU law», EU law beyond EU borders: the extraterritorial reach of EU law (Marise Cremona, Joanne Scott), (Oxford, Oxford University Press, 2019): 22-23. Joanne Scott, «New EU Extraterritoriality», The Common Market Law Review, 51, 5 (2014): 1355.

32 Reglamento del Parlamento Europeo y del Consejo, de 29 de abril de 2015, sobre el seguimiento, la notificación y la verificación de las emisiones de dióxido de carbono del transporte marítimo, (UE) 2015/757, DOEU 19.5.2015, L 123/55.

33 Como por ejemplo ocurre con la Directiva 2008/101 / CE del Parlamento Europeo y del Consejo, de 19 de noviembre de 2008, posteriormente comentada.

${ }^{34}$ Google Ireland Limited (Sociedad de capital registrada en Irlanda, cuyo domicilio social y lugar de administración central se encuentra en Dublín) demanda a Hungría por las multas que le impone, de conformidad a la «Ley de Impuesto sobre la Publicidad», con la que dicho Estado obliga a las empresas ubicadas en su Estado y en el resto del territorio europeo a la inscripción y al pago de impuestos, cuando éstas ofrezcan servicios por internet en húngaro, dándose por hecho que generalmente estos serán consumidos en dicho territorio. Por lo que la empresa publicitaria, registrada en otro Estado miembro, que ofrezca servicios por internet en húngaro ostentará las obligaciones extraterritoriales de comunicar los datos exigidos y de presentar las declaraciones tributarias requeridas. Y, en caso de incumplimiento, se le exigirá extraterritorialmente por parte de Hungría el pago de una multa proporcional a la responsabilidad que se genere. Por tanto, el propósito de la norma es suprimir las ventajas competitivas de las empresas de publicidad con gran volumen de negocio, a través de la concreción de obligaciones jurídicas en todo el territorio de la UE. No obstante en la práctica dicha norma afecta generalmente a las empresas ubicadas en el resto de los Estados miembros. Información extraída de CONCLUSIONES DE LA ABOGADO GENERAL SRA. JULIANE KOKOTT, presentadas el 12 de septiembre de 2019, Asunto Google Ireland Limited contra Nemzeti Adó- és Vámhivatal Kiemelt Adó- és Vámigazgatósága, C-482/18.

${ }^{35}$ Ley XXII de 2014 sobre la fiscalidad de los anuncios, «Ley sobre la fiscalidad de los anuncios» en la versión aplicable de 2016. 
para ofrecer servicios en una lengua minoritaria - el húngaro- sin que tenga presencia física en dicho Estado. Asimismo, considero que la conexión territorial es una característica principal porque aquellas exigencias de obligaciones extraterritoriales que se basen en la aplicación directa nunca presentarán dicha conexión como justificación de la acción referida, lo cual no exime de que evidentemente, existan otros tipos de nexos, temporales o nacionales.

En cualquier caso, la conexión territorial no será suficiente si no se complementa con otros requisitos, como la ineludible previsión y regulación de las medidas y efectos extraterritoriales que identifican los casos objeto de este trabajo. Mientras que en la aplicación directa del Derecho fuera de las fronteras, las respectivas medidas y efectos son causales y automáticos; en la modalidad de la extensión territorial, es completamente, al contrario, tanto las medidas como los consecuentes efectos se dispondrán minuciosa y previamente en la norma correspondiente siendo, por tanto, coercitivas. Esto es, generarán consecuencias, aunque solamente la norma será eficaz en el ámbito extraterritorial, si los sujetos internacionales implicados cooperan, ya que la imposición absoluta sería, evidentemente, una violación del principio de igualdad soberana, integridad territorial y no intervención. Así que al igual que se exige en la aplicación directa, todo ejercicio extraterritorial requerirá su congruencia con el Derecho Internacional y, por tanto, el respeto de la integridad territorial es una máxima jurídicamente incorruptible.

No obstante, ¿se puede entender que porque exista una conexión territorial una norma puede recoger cualquier tipo de medidas y efectos con incidencia más allá del propio territorio? Evidentemente, al igual que ocurre en la aplicación extraterriorial directa, por tanto, es una característica común, la extensión territorial se debe prever y aplicar en beneficio del interés general y común de toda la comunidad internacional. Por lo que, las distintas medidas que se exijan a otros Estados deberán respaldar y preservar estándares internacionales y valores comunes ${ }^{36}$ como la protección del medioambiente ${ }^{37}$,

36 «The principle of sovereignty and extraterritoriality cannot exempt the Community and the Commission from fulfilling the obligations attaching to that sovereignty to protect the general interest and act impartially, which undoubtedly include the obligation to establish, in accordance with a commitment made by the Commission, liabilities for events which endanger public safety, property and human lives, and to communicate the result of those investigations to interested parties who have sent claims for compensation to the Commission». Paragraph 40, Autosalone Ispra dei Fratelli Rossi/Comisión, Case C-136/01 P.

37 «The global commons and the global environment have been recognized as a «common concern» of humankind necessitating international cooperation for their protection but also reflecting an interest of the international community in their regulation». Sophia Kopela, «Port-State Jurisdiction, Extraterritoriality, and the Protection of Global Commons», Ocean Development \& International Law, 47, 2: 108. 
la salud pública, el derecho de los animales ${ }^{38}$, estabilidad y seguridad económica $^{39}$, etc., y no intereses exclusivamente propios.

Sin embargo, hasta las obligaciones que indudablemente estén escudadas en los principios de Naciones Unidas, amparadas en el principio de diligencia debida y que supongan intereses legítimos para toda la humanidad, se encontrarán limitadas por el principio de integridad territorial. Esto es, ningún Estado u Organización, como la UE, podrá exigir a otro igual la aplicación de disposiciones internas, vulnerando así su soberanía. La efectividad de la extraterritorialidad legítima solo podrá fundamentarse en la cooperación, de conformidad al Derecho Internacional.

\section{Confirmación de la teoría de la extensión territorial}

Como se viene exponiendo, la UE y sus Estados Miembros, huyendo de la aplicación directa extraterritorial del derecho, comienzan a través de la práctica a dar forma a otra modalidad de extraterritorialidad, con previsión en la norma, justificada y, por tanto, más segura jurídicamente, la cual la doctrina acaba calificando como extensión territorial y conceptualizando de conformidad a los parámetros descritos en el punto anterior.

Sin embargo, aunque la doctrina se pronuncie intensamente sobre dicha extensión territorial y, aunque cada vez en más ocasiones, la UE incluya la extraterritorialidad en sus normas, la realidad es que jurídica, oficial y tímidamente la confirmación de esta modalidad de aplicación extraterritorial no se produce hasta el 2011 por parte del TJUE. En este sentido, la Sentencia del TJUE que resuelve el sonado Asunto sobre el trasporte aéreo americano $^{40}$ avala, por primera y única vez en la historia, la legitimidad de la extensión de una norma más allá de las fronteras exteriores de la UE.

Sucintamente, los hechos más significativos son que, la UE en coherencia con su lucha y compromiso internacionalmente declarado por el cuidado del medioambiente y, en concreto, para reducir las emisiones de gases de efecto invernadero, adoptó en 2008 la Directiva para incluir las actividades de aviación en el régimen comunitario de comercio de derechos de emisión de gases de efecto invernadero ${ }^{41}$ - en adelante Directiva sobre aviación-,

38 Reglamento del Consejo, de 24 de septiembre de 2009, sobre la protección de los animales en el momento de la matanza, (CE) no 1099/2009, DOEU 18.11.2009, L 303/1.

39 Véase los ejemplos referenciados en la nota 30.

40 Sentencia del Tribunal de Justicia (Gran Sala) de 21 de diciembre de 2011, Asunto C-366/10 sobre la asociación de transportistas aéreos de América.

${ }^{41}$ Directiva 2008/101/CE del Parlamento Europeo y del Consejo, de 19 de noviembre de 2008 , por la que se modifica la Directiva 2003/87/CE con el fin de incluir las actividades de 
la cual exige como regla general, que todas las aerolíneas que despeguen o aterricen en territorio de la Unión deberán facilitar a la organización aquellos datos sobre las emisiones que sean solicitados. Exceptuándose de dicha obligación a aquellos terceros Estados que tienen sus propios planes de control medioambiental o que adoptan algún acuerdo para preservar el objetivo descrito. En este sentido, la Directiva sobre aviación establece la obligación extraterritorial para aquellas compañías aéreas cuyos vuelos tengan recepción o salida desde el territorio de cualquiera de los Estados Miembros, de facilitar datos relativos a la producción de gases de efecto invernadero que tiene su actividad. Así las cosas, la Directiva europea extiende a compañías aéreas y, en última instancia, a terceros Estados que quieran tener contacto territorial y directo, a que notifiquen sus emisiones, con la pretensión de limitar unilateralmente el volumen de tráfico o incluso frecuencia o periodicidad de los servicios aéreos, porque se superen los límites que se estiman recomendables ${ }^{42}$. Y en este respecto, destacan dos elementos jurídicamente llamativos; en primer lugar, como se viene comentando, se extiende a un tercer sujeto el cumplimiento de una obligación, la cual logra eficacia y que no se viole el principio de soberanía si existe cooperación, es decir, no cabe la imposición absoluta de su cumplimiento. En segundo lugar, es llamativo tener presente que, se satisfaga o no la imposición, la propia UE tiene facultad en el marco de sus competencias de limitar unilateralmente el vínculo con el otro sujeto o Estado que cumple o no la norma en cuestión. Por tanto, podemos percibir el principio de soberanía territorial en todo su esplendor; cuando unilateralmente restringe conexiones porque choca con su núcleo de obligaciones e intereses (con su poder territorial) y, cuando se exceptúa su rigidez para que, mediante la colaboración, los sujetos internacionales logren acomodar sus inmensos ordenamientos jurídicos.

En cualquier caso, el objetivo de este apartado es poder destacar que si la referida sentencia del TJUE sigue dando de qué hablar es porque, indudablemente, con ella se consigue confirmar la singular teoría de la extensión territorial. Si ya paulatinamente la UE en su conjunto iba definiendo su posición de la extraterritorialidad con ciertas normas y prácticas cada vez más atrevidas, se puede decir que la UE definitivamente «sale del armario» al exigir que las líneas aéreas, como las americanas o chinas, se comprome-

aviación en el régimen comunitario de comercio de derechos de emisión de gases de efecto invernadero, DOUE 13.1.2009, L 8/3.

42 Artículo 15 de la Directiva 2008/101/CE del Parlamento Europeo y del Consejo, de 19 de noviembre de 2008, por la que se modifica la Directiva 2003/87/CE con el fin de incluir las actividades de aviación en el régimen comunitario de comercio de derechos de emisión de gases de efecto invernadero. 
tan a rendir cuentas, como si de un hermano mayor se tratara. Por tanto, era evidente la disconformidad que presentarían algunos Estados frente a estas obligaciones y esto acaba ocasionando el estudio de la referida Directiva y el irremediable fallo por el Tribunal de la Unión.

En mi opinión, el Tribunal de Justicia respaldando la valentía que demuestra la UE al exigir que el resto de la comunidad internacional se comprometa con cuestiones tan controvertidas como el respeto al medioambiente, es riguroso al analizar cada uno de los requisitos que identifica la figura de la extensión territorial y termina avalando directamente sus elementos y, por tanto, a la figura de la extensión territorial que la Directiva recoge en sus disposiciones.

En primer lugar, ampara y protege que el objetivo de la Directiva sobre Aviación, que es en sentido último la protección del medioambiente, corresponde a un objetivo claramente global, que se impulsa por el Derecho Internacional Público ${ }^{43}$ y que, como es de general conocimiento, es un objetivo de la propia UE ${ }^{44}$, así como de sus Estados Miembros. Por lo que sería insostenible que aquel Estado no comprometido con el mismo, en situación minoritaria, aludiera que el fundamento de dicha obligación que sobrepasa las fronteras se apoya en un interés privado u oculto para la UE.

En segundo lugar, como se viene explicando con detalle, el Tribunal tiene que admitir que la obligación que se extiende hacia dichas aeronaves, viene específicamente recogida en la Directiva ${ }^{45}$. Por lo que ni las medidas para el control de las emisiones, ni que la disposición tendrá efectos ad extra, pueden ser calificadas como casuales e inesperadas.

Y, en último lugar y tal vez con mayor importancia, el TJUE reconoce que, manifiestamente, el deber que deriva de la Directiva en cuestión se

43 Parece innecesario aclara que anualmente los líderes y expertos de los Estados de toda la comunidad internacional se reunen en la Conferencia de las Naciones Unidas sobre Cambio Climático tal y como recoge la Convención Marco de las Naciones Unidas sobre el Cambio Climático. El último gran tratado internacional adoptado y firmado casi por unanimidad fue el Acuerdo de París de 2015.

${ }^{44}$ En el Tratado de la Unión Europea se recoge en el preámbulo y en los artículos 3.3. y 21.2.d y f.

45 «Dichos principios del Derecho consuetudinario internacional se invocan, en esencia, para que el Tribunal de Justicia aprecie si la Unión era competente, en relación con dichos principios, para adoptar la Directiva 2008/101, pues ésta extiende la aplicación de la Directiva 2003/87 a los operadores de aeronaves de terceros Estados cuyos vuelos con destino u origen en un aeródromo situado en el territorio de un Estado miembro de la Unión se realicen en parte sobre la alta mar y sobre el territorio de éstos». Sentencia del Tribunal de Justicia (Gran Sala) de 21 de diciembre de 2011, Asunto C-366/10 sobre la asociación de transportistas aéreos de América, párrafo 108. 
ampara en una clara conexión territorial ${ }^{46}$; los vuelos que despeguen o aterricen en los Estados Miembros de la $\mathrm{UE}^{47}$. Y, por tanto, no se exigirá la obligación a los «vuelos internacionales que sobrevuelan el territorio de los Estados miembros de la Unión o el de terceros Estados» ${ }^{48}$.

En definitiva, la obligación establecida en la Directiva no es de interés particular, al fundamentarse en el Derecho Internacional y al ser de común beneficio, no solo es proporcional, sino que cabe que se exija extraterritorialmente. De manera que, la norma es clara con el supuesto de hecho, los efectos y las consecuencias Y, por tanto, solo queda que los sujetos implicados, voluntariamente, cumplan con el requerimiento que la UE extiende hasta ellos. Claro que están legitimados para hacer oídos sordos, pero la UE - si le acaba conviniendo para sus otros intereses - podrá cumplir y unilateralmente «tomar nota».

\section{Conclusiones}

- La primera conclusión que se puede extraer, incluso atendiendo simplemente al índice del trabajo, es que la extraterritorialidad es un fenómeno que va perdiendo excepcionalidad, aunque al mismo tiempo su aplicación requiera un equilibrio que lo convierte en una difícil tarea. Si algo es evidente, es que la distinción entre

46 «It actually rests upon a territorial trigger as it only applies to flights arriving at or departing from an EU airport». Joanne Scott, «Extraterritoriality and Territorial Extension of EU Law», American Journal of Comparative Law, 62, 1 (2014): 97.

47 «A este respecto, la Directiva 2008/101 modificó el anexo I de la Directiva 2003/87, insertando en el mismo una categoría de actividad titulada «Aviación» y añadiendo en el punto 2 de la introducción a dicho anexo un segundo párrafo conforme al cual, «a partir del 1 de enero de 2012, se incluirán todos los vuelos con destino u origen en un aeródromo situado en el territorio de un Estado miembro al que se aplica el Tratado». 116 Sobre este aspecto, ha de señalarse que, entre las exclusiones enumeradas en dicho anexo I, no figuran criterios vinculados, por lo que respecta a las aeronaves que salen de un aeródromo de la Unión, al aeródromo de llegada ni, para las aeronaves que llegan a un aeródromo de la Unión, al aeródromo de salida. En consecuencia, la Di rectiva 2008/101 se aplica indistintamente a los vuelos con destino o procedencia del territorio de la Unión, incluidos los procedentes o con destino a aeródromos situa dos fuera de este territorio. También resulta así del decimosexto considerando de la Directiva 2008/101». Sentencia del Tribunal de Justicia (Gran Sala) de 21 de diciembre de 2011, Asunto C-366/10 sobre la asociación de transportistas aéreos de América, párrafos 115-116.

48 «Por tanto, dicha Directiva no pretende aplicarse como tal a vuelos internacionales que sobrevuelan el territorio de los Estados miembros de la Unión o el de terceros Estados, cuando tales vuelos no tengan destino u origen en un aeródromo situado en el territorio de un Estado miembro». Sentencia del Tribunal de Justicia (Gran Sala) de 21 de diciembre de 2011, Asunto C-366/10 sobre la asociación de transportistas aéreos de América, párrafo 117. 
territorialidad y extraterritorialidad, especialmente en la práctica, no está claramente delimitada.

- Si la aplicación extraterritorial del derecho es compleja, ¿por qué en los últimos tiempos se utiliza con mayor frecuencia? La respuesta es sencilla, se debe a que, como cada vez los ordenamientos jurídicos son más densos y espaciosos, para que todos los Estados puedan tener una cómoda estancia en la comunidad internacional institucionalizada, la extraterritorialidad se contempla como una opción cada vez más factible, que permite no quedase atrás o lo que es lo mismo, exigir a otro sujeto el cumplimiento de cuestiones relevantes para el primero.

- La extraterritorialidad no es un «duty free» de los sagrados principios del derecho, sino que se exceptúa relativamente el principio territorial porque el sujeto internacional receptor de la obligación, admite su deber a través de la cooperación.

- Así las cosas, aunque el ejercicio extraterritorial «puro y duro» nunca ha sido del agrado de la Unión, cada vez en mayor medida los Estados Miembros y la UE comienzan a recoger distintas cláusulas en las que se contemplan efectos extraterritoriales, aunque la intención (al menos en teoría) no sea la de imponer intereses privados u ocultos.

- Como muestra la práctica, la UE en general rehúye de la aplicación directa de normas más allá de las fronteras, pero sí que parece sentirse cómoda exigiendo ciertas disposiciones a través de la extensión territorial y así dar coherencia a sus obligaciones en un mundo globalizado.

- La extensión territorial consiste en la aplicación de una norma, la cual tiene como objetivo respaldar un interés común, más allá de las fronteras, porque se produce una conexión territorial entre el sujeto que regula la medida y el que, voluntariamente, cumple con los efectos esperados. Dichos rasgos son conceptualizados por la doctrina, tras observarse distintas prácticas tímidamente extraterritoriales que presentan un patrón común.

- En este sentido, la confirmación de dicha modalidad de extraterritorialidad no se produce hasta que la indignación de las aeronaves americanas provoca que el propio TJUE se pronuncie sobre la legalidad de una norma jurídica vinculante que les exigía el cumplimiento de ciertas tareas. Y, así las cosas, aunque el alto tribunal avala la norma en cuestión y, por tanto, confirma la teoría de la extensión territorial, la Comisión Europea no ha hecho efectivas todas las obligaciones que jurídicamente emanaban de la comentada Directiva sobre Aviación. 


\section{Sobre la autora}

Ana Cristina Gallego Hernández es Doctora en Derecho con mención internacional, Máster en Derecho Público y Licenciada en Derecho por la Universidad de Sevilla, así mismo, es Diplomada por la Academia de Derecho Internacional de la Haya, en el Centro de Estudios e Investigación, en 2019. Es profesora, acreditada como Contratado Doctor, de Derecho Internacional Público y Relaciones Internacionales en el Centro Universitario San Isidoro (adscrito a la Universidad Pablo de Olavide). Como miembro del equipo de investigación Política y Derecho Internacional (SEJ119), sus principales líneas de investigación engloban: tratados internacionales, seguridad sanitaria, paz y seguridad internacionales y extraterritorialidad. Ha participado en diversos Proyectos Nacionales e Internacionales y es autora de más de una decena de publicaciones en las que destaca la monografía, fruto de su tesis doctoral, El Derecho Internacional a la Salud a la luz de la Paz y Seguridad Internacionales, galardonada con el II Premio Eduardo de Hinojosa y Naveros en Ciencias Jurídicas.

\section{About the autor}

Ana Cristina Gallego Hernández is a $\mathrm{PhD}$ in Law (international mention), a Master's in Public Law and a Law Degree at the University of Seville. Also, she is a Diploma in the Center for Studies and Research at the Hague Academy of International Law, in 2019. She is an associate professor, accredited as a Contracted Lecturer, of International Public Law and International Relations at the San Isidoro University Center (attached to the Pablo de Olavide University). As a member of the Political and International Law research group (SEJ119), her main lines of research encompass: international treaties, health security, international peace and security and extraterritoriality. She has participated in different National and International Projects and is the author of more than a dozen publications, among which the monograph stands out (as result of her doctoral thesis): El Derecho Internacional a la Salud a la luz de la Paz y Seguridad Internacionales, awarded with the II Eduardo de Hinojosa y Naveros Prize in Legal Sciences. 


\section{Derechos de autor}

Los derechos de autor (para la distribución, comunicación pública, reproducción e inclusión en bases de datos de indexación y repositorios institucionales) de esta publicación (Cuadernos Europeos de Deusto, CED) pertenecen a la editorial Universidad de Deusto. El acceso al contenido digital de cualquier número de Cuadernos Europeos de Deusto es gratuito inmediatamente después de su publicación. Los trabajos podrán leerse, descargarse, copiar y difundir en cualquier medio sin fines comerciales y según lo previsto por la ley; sin la previa autorización de la Editorial (Universidad de Deusto) o el autor. Así mismo, los trabajos editados en CED pueden ser publicados con posterioridad en otros medios o revistas, siempre que el autor indique con claridad y en la primera nota a pie de página que el trabajo se publicó por primera vez en CED, con indicación del número, año, páginas y DOI (si procede). Cualquier otro uso de su contenido en cualquier medio o formato, ahora conocido o desarrollado en el futuro, requiere el permiso previo por escrito del titular de los derechos de autor.

\section{Copyright}

Copyright (for distribution, public communication, reproduction and inclusion in indexation databases and institutional repositories) of this publication (Cuadernos Europeos de Deusto, CED) belongs to the publisher University of Deusto. Access to the digital content of any Issue of Cuadernos Europeos de Deusto is free upon its publication. The content can be read, downloaded, copied, and distributed freely in any medium only for non-commercial purposes and in accordance with any applicable copyright legislation, without prior permission from the copyright holder (University of Deusto) or the author. Thus, the content of CED can be subsequently published in other media or journals, as long as the author clearly indicates in the first footnote that the work was published in CED for the first time, indicating the Issue number, year, pages, and DOI (if applicable). Any other use of its content in any medium or format, now known or developed in the future, requires prior written permission of the copyright holder. 